POS $\quad$ PROCEEDINGS

\title{
Study of the variable broadband emission of Markarian 501 during the most extreme Swift X-ray activity
}

\section{J. Becerra González*,}

Universidad de La Laguna, Dpto. Astrofísica, E-38206 La Laguna, and Inst. de Astrofísica de Canarias, E-38200 La Laguna, Tenerife, Spain

E-mail: jbecerragonzalez@gmail.com

D. Paneque

Max-Planck-Institut für Physik, D-80805 München, Germany

C. Wendel

Universität Würzburg, D-97074 Würzburg, Germany

F. Tavecchio

Osservatorio di Brera, Milan and National Institute for Astrophysics (INAF), I-00136 Rome, Italy

K. Noda

ICRR, The University of Tokyo, 277-8582 Chiba, Japan

K. Ishio

Max-Planck-Institut für Physik, D-80805 München, Germany

\section{J. Sitarek}

University of Łódź, Department of Astrophysics, PL-90236 Łódź, Poland

for the MAGIC'and Fermi-LAT Collaborations

For collaboration list see PoS(ICRC2019)1177 
Markarian 501 (Mrk 501) is a bright very-high-energy (VHE, E>100 GeV) BL Lac object. Thanks to its brightness and its proximity $(\mathrm{z}=0.034)$, it can be detected in the VHE even during low states, making it an excellent blazar for unbiased (not dependent on the emission state) multi-wavelength (MWL) studies. During a multi-wavelength campaign in 2014 (including data from VHE to the radio band: MAGIC, FACT, Fermi-LAT, Swift, KVA and Metsähovi), Mrk 501 displayed the highest X-ray activity observed by Swift-XRT since its launch more than 14 years ago.

The flare lasted for a 2-week period in July 2014, during which the source is clearly detected in both X-rays and the VHE band as observed by MAGIC and FACT telescopes. The X-ray spectra are very hard and show variability on nightly timescales in X-rays and the VHE band. A correlation between these two energy bands was found. During the 2-week long flaring episode, Mrk 501 was detected at a VHE flux level above $150 \mathrm{GeV}$ between 0.2 to 2 times the Crab Nebula flux. Thanks to the rich (quasi-)simultaneous MWL data sample, nightly MWL spectral energy distributions (SEDs) were built. The temporal evolution of the nightly SEDs can be described by a one-zone synchrotron self-Compton (SSC) model with variations in the break energy of the electron energy distribution and its shape, as well as some small variations in the magnetic field strength within the relativistic jet. Therefore, the flux evolution during the flare can be explained in terms of acceleration and the cooling of the high-energy electrons.

During the peak flux measured in X-rays above $0.3 \mathrm{keV}$, 2014 July 19 (MJD 56857.98), a narrow feature centered around $3 \mathrm{TeV}$ was observed in the VHE spectrum observed by the MAGIC telescopes. Such spectral feature is not compatible with the classical analytic function used to describe the VHE blazar spectra as power-law, log-parabola and log-parabola with an exponential cut-off at more than $3 \sigma$ confidence level (C.L.). A likelihood ratio test (LRT) results in a preference at about $4 \sigma$ C.L. of a log-parabola plus a narrow component w.r.t. a single log-parabola. In addition, a Monte Carlo simulation was performed to estimate the significance of the existence of such extra component following the prescriptions from [1]. The result is also consistent with the previous tests, finding a significance at a level of $3 \sigma$ C.L. for an extra narrow spectral component in addition to a log-parabola (null hypothesis). Assuming that such feature is real, three different theoretical scenarios have been investigated to reproduce the MWL SED including this additional narrow spectral feature: a) a pile-up in the electron energy distribution due to stochastic acceleration; b) a structured jet including two-zone SSC model; and c) a magnetospheric vacuum gap model in addition to one-zone SSC framework.

36th International Cosmic Ray Conference -ICRC2019-

July 24th - August 1st, 2019

Madison, WI, U.S.A.

\footnotetext{
*Speaker.

†https://magic.mpp.mpg.de/
} 


\section{Acknowledgements}

We would like to thank the Instituto de Astrofísica de Canarias for the excellent working conditions at the Observatorio del Roque de los Muchachos in La Palma. The financial support of the German BMBF and MPG, the Italian INFN and INAF, the Swiss National Fund SNF, the ERDF under the Spanish MINECO (FPA2015-69818-P, FPA2012-36668, FPA2015-68378-P, FPA2015-69210-C6-2-R, FPA2015-69210-C6-4-R, FPA2015-69210-C6-6-R, AYA2015-71042-P, AYA2016-76012-C3-1-P, ESP2015-71662-C2-2-P, FPA2017?90566?REDC), the Indian Department of Atomic Energy, the Japanese JSPS and MEXT, the Bulgarian Ministry of Education and Science, National RI Roadmap Project DO1-153/28.08.2018 and the Academy of Finland grant nr. 320045 is gratefully acknowledged. This work was also supported by the Spanish Centro de Excelencia "Severo Ochoa" SEV-2016-0588 and SEV-2015-0548, and Unidad de Excelencia "María de Maeztu" MDM-2014-0369, by the Croatian Science Foundation (HrZZ) Project IP2016-06-9782 and the University of Rijeka Project 13.12.1.3.02, by the DFG Collaborative Research Centers SFB823/C4 and SFB876/C3, the Polish National Research Centre grant UMO2016/22/M/ST9/00382 and by the Brazilian MCTIC, CNPq and FAPERJ.

The Fermi LAT Collaboration acknowledges generous ongoing support from a number of agencies and institutes that have supported both the development and the operation of the LAT as well as scientific data analysis. These include the National Aeronautics and Space Administration and the Department of Energy in the United States, the Commissariat à l'Energie Atomique and the Centre National de la Recherche Scientifique / Institut National de Physique Nucléaire et de Physique des Particules in France, the Agenzia Spaziale Italiana and the Istituto Nazionale di Fisica Nucleare in Italy, the Ministry of Education, Culture, Sports, Science and Technology (MEXT), High Energy Accelerator Research Organization (KEK) and Japan Aerospace Exploration Agency (JAXA) in Japan, and the K. A. Wallenberg Foundation, the Swedish Research Council and the Swedish National Space Board in Sweden.

Additional support for science analysis during the operations phase is gratefully acknowledged from the Istituto Nazionale di Astrofisica in Italy and the Centre National d'Études Spatiales in France. This work performed in part under DOE Contract DE-AC02-76SF00515.

J. Becerra González acknowledges the support of the Viera y Clavijo program funded by ACIISI and ULL.

\section{References}

[1] Tombesi, F., Cappi, M., Reeves, J. N., et al. 2010, A\&A, 521, A57 